\title{
THIN FILMS WITH HIGH SURFACE TENSION*
}

\author{
T. G. MYERS ${ }^{\dagger}$
}

Abstract. This paper is a review of work on thin fluid films where surface tension is a driving mechanism. Its aim is to highlight the substantial amount of literature dealing with relevant physical models and also analytic work on the resultant equations.

In general the introduction of surface tension into standard lubrication theory leads to a fourthorder nonlinear parabolic equation

$$
\frac{\partial h}{\partial t}+\frac{\partial}{\partial x}\left(C \frac{h^{3}}{3} \frac{\partial^{3} h}{\partial x^{3}}+f\left(h, h_{x}, h_{x x}\right)\right)=0,
$$

where $h=h(x, t)$ is the fluid film height. For steady situations this equation may be integrated once and a third-order ordinary differential equation is obtained. Appropriate forms of this equation have been used to model fluid flows in physical situations such as coating, draining of foams, and the movement of contact lenses.

In the introduction a form of the above equation is derived for flow driven by surface tension, surface tension gradients, gravity, and long range molecular forces. Modifications to the equation due to slip, the effect of two free surfaces, two phase fluids, and higher dimensional forms are also discussed. The second section of this paper describes physical situations where surface tension driven lubrication models apply and the governing equations are given. The third section reviews analytical work on the model equations as well as the "generalized lubrication equation"

$$
\frac{\partial h}{\partial t}+\frac{\partial}{\partial x}\left(h^{n} h_{x x x}\right)=0
$$

In particular the discussion focusses on asymptotic results, travelling waves, stability, and similarity solutions. Numerical work is also discussed, while for analytical results the reader is directed to existing literature.

Key words. lubrication theory, thin films, surface tension, fourth-order diffusion equations

AMS subject classifications. 35-02, 35A20, 35B40, 35G20, 35K55, 35K65, 76-02, 76B45, $76 \mathrm{D} 08$

PII. S003614459529284X

1. Introduction. The lubrication or reduced Reynolds number approximation to the Navier-Stokes equations has been used to describe a multitude of situations. Recently attention has been focussed on the situation where surface tension plays an important role, of which rain running down a window, the evolution of drying paint layers, or the spreading of a fluid drop on a surface are all examples; see [50].

Typically surface tension driven thin film flows are described by a fourth-order nonlinear degenerate parabolic equation of the form

$$
\frac{\partial h}{\partial t}+\frac{\partial}{\partial x}\left\{\frac{h^{3}}{3}\left(C h_{x x x}-\delta B h_{x} \cos \alpha+B \sin \alpha\right)+A \frac{h_{x}}{h}+\frac{M}{2} \sigma_{x} h^{2}\right\}=0,
$$

where $h(x, t)$ is the film height, $\delta$ is the aspect ratio (typical height/typical length scale), $\alpha$ is the angle of inclination of the flow, and subscripts denote differentiation with respect to that variable. The configuration is shown in Figure 1. Equation (1) shows that fluid film height varies due to the effect of surface tension, gravity, long range molecular forces, and surface tension gradients. The relative strength of each term is reflected in the coefficients: $C$, the inverse capillary number, is the ratio

${ }^{*}$ Received by the editors October 4, 1995; accepted for publication (in revised form) July 15, 1997. http://www.siam.org/journals/sirev/40-3/29284.html

†OCIAM, Mathematical Institute, 24-29 St Giles', Oxford, OX1 3LB (t.g.myers@cranfield.ac.uk). 


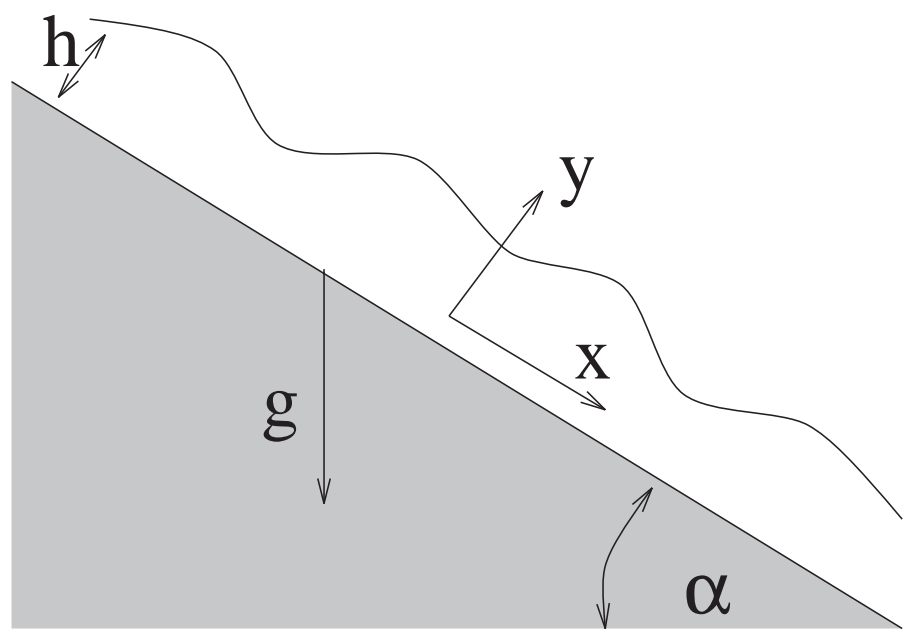

FIG. 1. A thin fluid film on an inclined plane.

of surface tension to viscous forces; $B$, the Bond number, is the ratio of gravity to viscous forces; $A$ is a van der Waals term; and $M$, the Marangoni number, is the ratio of surface tension gradients to viscous forces. Equation (1) is derived in the appendix.

In two dimensions the lubrication equation takes the form

$$
h_{t}+\nabla \cdot\left[\frac{h^{3}}{3}\left(C \nabla \nabla^{2} h-\delta B \nabla h \cos \alpha+B \mathbf{r} \sin \alpha\right)+A \frac{\nabla h}{h}+\frac{M}{2} h^{2} \nabla \sigma\right]=0 .
$$

Perhaps the most widely used steady version of (1) is

$$
C h^{3} h_{x x x}-3 U h=-3 U h_{\infty},
$$

where $h_{\infty}$ is the equilibrium film thickness. This is known as the Landau-Levich equation and was originally derived in [42] to determine the thickness of the coating when cinefilm is pulled out of a bath with constant velocity $U$. It has subsequently been derived to describe the thickness of a soapfilm [51], the wetting layer when a bubble moves through a capillary tube [19], the height of a tear film around a contact lens [47], and flow at the interface between a strip under tension and a coating roller [57].

In the derivation of (3) surface tension is the only driving force; the no-slip condition on a moving substrate introduces the $-U h$ term (a systematic derivation is given in [73]). Alternatively, a travelling wave substitution $h(x, t)=H(x-U t)$ will reduce (1) to (3) with an appropriate choice for the constant of integration and $A$ and $B$ set to zero. The solution of (3) is discussed in section 3.2.

Situations where the film thickness reaches zero, such as at the front of a moving drop or when a film breaks up, introduce the added complication of contact angles and a moving contact line. The contact angle and contact line motion are properties inherent to each particular system and depend on properties such as the wetting and dewetting fluid composition, surface chemistry, and surface roughness. As yet no theory has been developed to adequately describe the phenomena. A full discussion of contact line motion is beyond the scope of this review and the reader is referred to [25], [26], [30], and [43], which provide a wealth of information on the subject. 
Experimental results on contact angle versus contact line speed show the contact angle increasing with speed. However, when the velocity is zero a jump will occur between the values of the static contact angle measured from a receding system to an advancing one. This nonuniqueness is known as contact angle hysteresis and occurs on all but the smoothest surfaces; see [25]. In the regions where the velocity is nonzero it is commonly accepted that the contact line velocity may be adequately related to the dynamic contact angle via "Tanner's law"

$$
\tan \theta_{d} \propto U^{1 / 3},
$$

where the constant of proportionality will be determined by the particular system [69].

When modelling a dynamic problem the standard no-slip condition requires an infinite stress to move the contact line; any slip condition (i.e., $u=f(h) u_{y}$, where $u$ is the fluid velocity in the $x$-direction) will alleviate this problem [26]. In practice the most common slip laws used are

$$
\text { (a) } u=\frac{\alpha}{3 h} u_{y} \quad \text { or } \quad \text { (b) } \quad u=\beta u_{y},
$$

where $\alpha$ and $\beta$ are constant slip coefficients. This will modify the coefficient $h^{3}$ in (1) and (2) to $\left(h^{3}+a h^{m}\right)$, where $m$ takes the value 1 or 2 for models (a) and (b), respectively. The coefficient $a \ll 1$, so that this extra term is negligible everywhere except for in the vicinity of the contact line, where $h^{3-m}$ is $O(a)$. Models where this extra term, with $m<3$, dominates are discussed in section 3 .

That a front may move without slipping can be seen from analogies of a carpet unrolling or a rolling ball. Hence, an alternative approach for modelling a dynamic contact line is to formalize the concept of fluid rolling in the vicinity of this line. This was suggested in [26] together with convincing experimental evidence. The problem is investigated analytically in [65].

Certain fluids have been observed to shoot out a "precursor film" ahead of the bulk fluid [3], and this leads to another method for dealing with moving contact lines. Mathematically this signifies that a calculation may be terminated when the film thickness reaches an empirically determined value (see section 2.1 and [70]). Alternatively, the problem may be formulated in such a way that as $h \rightarrow 0$ long range molecular forces become significant and thus permit the film thickness to reach zero; this is discussed in section 2.6.

Equations of the form (1) and (2) apply if the fluid motion is constrained by a no-slip, or similar, condition on one surface, producing a shear flow. When the fluid has two free surfaces, as with a soapfilm, the basic flow is "extensional" and such equations do not, in general, apply. Because the fluid velocity is now unknown on both surfaces a higher order approximation in $\delta$ is generally required to close the leading order problem. This leads to

$$
\begin{aligned}
h_{t}+(u h)_{x} & =0, \\
\left(4 h u_{x}\right)_{x}+C h h_{x x x}+B h \sin \alpha & =0 .
\end{aligned}
$$

This system is derived in [27], [34]. To arrive at a simpler problem many authors assume one surface to be loaded with surfactant, meaning it may be treated as inextensible. The fluid velocity on this surface may then be specified (and is generally set to zero) reducing the problem to (1).

The aim of this present work is to itemize the wide variety of applications of lubrication equations such as (1) and (2) and also to review relevant mathematical 
results. In section 2 a list of physical situations, where (1) or related equations have been used to describe the flow, is given. It is arranged by starting with the simplest flows, on a solid substrate, driven solely by gravity and surface tension. Surface tension gradients and long range molecular force models are subsequently introduced and finally models where the fluid is not constrained by a substrate, such as foams, are discussed. In section 3 relevant mathematical results and techniques are described. Again the simplest problems are considered first, hence steady state results are reviewed in the early sections; asymptotic results and travelling wave analyses are then described. These are followed by stability analyses, similarity solutions, and unsteady numerical work. Analytical results on the generalized lubrication equation, given in section 3.1, are also briefly discussed, but due to the vast number of results the reader is referred to more appropriate texts.

\section{Physical processes.}

2.1. Coating. Coating flows involve covering a surface with one or more thin layers of fluid. They range from rain running down a window to manufacturing processes, such as the production of videotapes. Difficulties in modelling coating flows arise for a number of reasons. For example, operating conditions may require a running speed which leads to instabilities, such as air entrainment and ribbing (a stationary surface wave perpendicular to the direction of flow) [55], [61]. Fluid rheology may also be complex. Thus a great number of coating devices have been developed to accommodate the variety of applications and operating conditions. They may be separated into three main categories.

i) Free coating. Examples include spin coating, where a fluid spreads over a spinning surface [58] and dip coating [42], [73].

ii) Metered coating, where an obstacle is placed in order to limit the thickness of coating on the substrate. Examples include blade coating and roll coating [73], [29].

iii) Transfer coating, where a uniform film is transferred onto a moving substrate. Examples are slot, slide/curtain [28], and also gravure coating.

Other coating methods exist. For example, a recent method used in the paint industry is to charge paint particles while spraying them onto an earthed workpiece. The system is then heated, so the particles sinter forming a uniform layer. These "solid" coating methods will not be discussed in the present review. Further reviews on coating flows may be found in [4], [5], [28], and [60].

A lubrication model for surface tension driven flow on a curved substrate is developed in [64]. This gives rise to a variant of (1) in the form

$$
h_{t}+\frac{\partial}{\partial s}\left(C \frac{h^{3}}{3}\left(h_{s s s}+\kappa_{s}\right)\right)=0,
$$

where $s$ is the coordinate tangential to the substrate and $\kappa$ is the curvature. More general models may be found in [35], [38].

In [70] a number of autonomous ordinary differential equations, related to draining flow down a vertical wall, are studied. A typical situation is shown in Figure 2, where a fluid is draining down a dry wall. It may be modelled by

$$
h_{x x x}=-1+\frac{1}{h^{2}},
$$

where the third-order term represents surface tension, the constant term models gravity, and the $h^{-2}$ term comes from viscous shearing forces. The film thickness and $x$ have been appropriately scaled to remove the coefficients. 


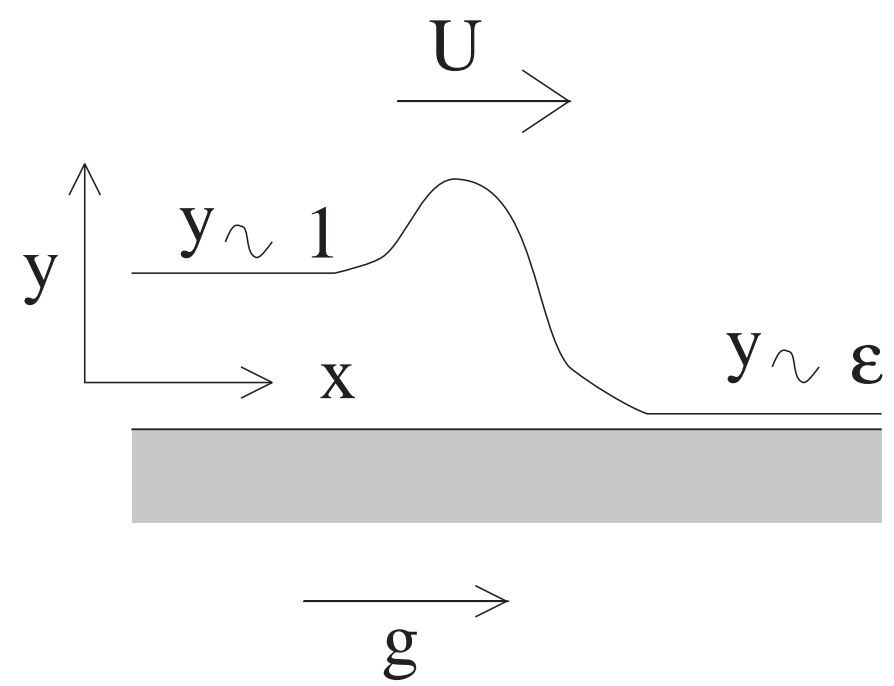

FiG. 2. Vertical draining flow.

Problems associated with the contact line, where $h=0$, provide the incentive for studying a number of other equations describing inner and outer expansions in the vicinity of the contact line. In particular

$$
h_{x x x}=-1+\frac{1+\epsilon+\epsilon^{2}}{h^{2}}-\frac{\epsilon+\epsilon^{2}}{h^{3}}
$$

describes draining in the presence of a precursor fluid layer of thickness $\epsilon$, as shown in Figure 2. As $\epsilon \rightarrow 0$ this reduces to (8). Other equations dealt with include a scaled form of (3) which is the limit of (9) for $h=O(\epsilon)$ as $\epsilon \rightarrow 0$ and

$$
h_{x x x}=h^{-2}
$$

which is the intermediate limit, being the small $h$ limit of (8) and the large $h$ limit of (3). They also describe two model equations which allow slip and demonstrate numerically that slip models and models with a precursor layer generate almost identical results, provided the slip coefficient is within a certain carefully chosen range.

2.2. Condensate motion on heat exchangers. Film condensation is a method of heat transfer used in chemical plants, refrigerators, air conditioners, and other cooling devices. The method of cooling involves passing a vapor over metal fins which are kept below the condensation temperature. Condensate builds up on the fins, flows into the channel between them, and subsequently drains away. At low vapor flow rates the transfer of heat to the fins has been found to be much enhanced compared to measurements at higher flow rates. Surface tension aided drainage, producing locally thin films and thus reducing thermal resistance, has been proposed as the mechanism responsible for this discrepancy [32]. Figure 3 shows a typical cooling fin configuration.

At the top of the fin large stresses, resulting from the high curvature, act to reduce film thickness. Over the central region of a fin, curvature is low and gravity dominates, while in the trough high curvature produces a suction effect which acts to pull fluid down the side wall and increases the draining rate. 


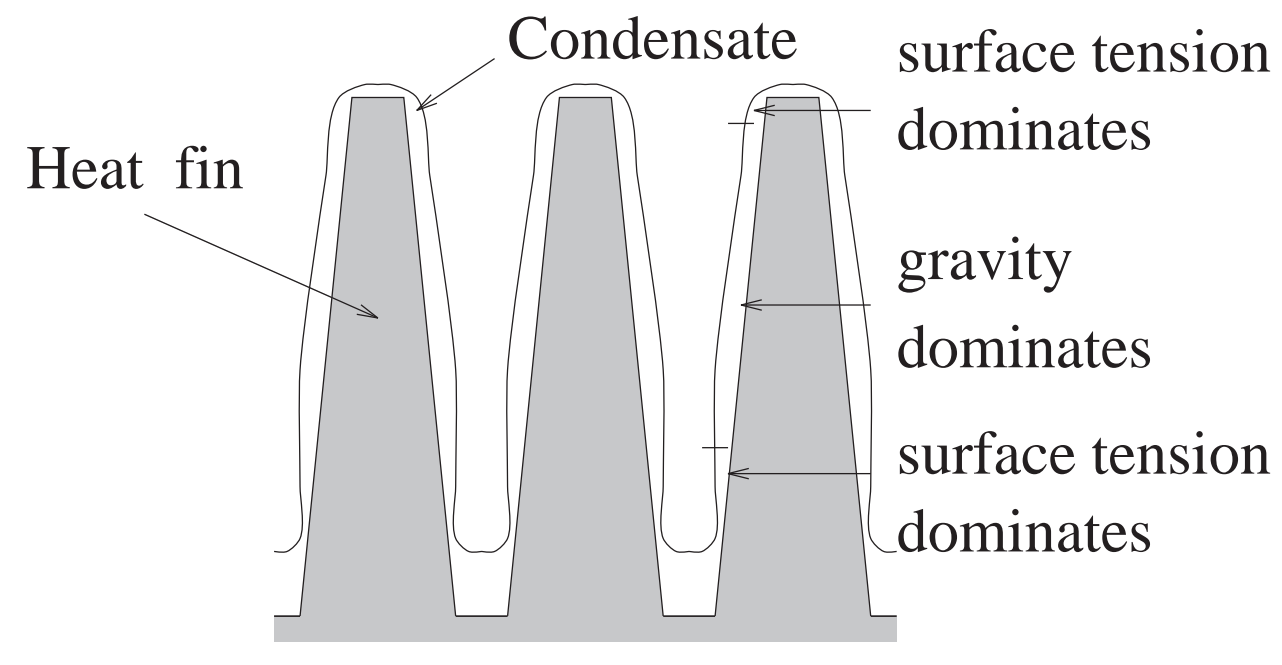

Fig. 3. Cooling fin configuration.

Provided gravity forces are small in comparison to surface tension forces, for example near the base or top of a fin, the governing equation for film height is

$$
\left(C h^{3} h_{x x x}\right)_{x}=\frac{\gamma}{h} \Delta T,
$$

where $\Delta T$ is the difference in temperature between the vapor and fin and $\gamma$ is constant. This final term follows from a simple model in which condensate buildup on the fins is proportional to $\Delta T$.

Analysis shows that a sharp fin tip provides the best drainage in this region. Around the trough, noting that the geometry is equivalent to that of dip coating, the method of [42] may be used to determine the film thickness. Numerical results show that the film becomes very thin above the trough and this local thinning, due to suction, combined with optimizing the curvature at the top of a fin can improve heat transfer by up to three times that predicted by Nusselt theory [22], [32].

2.3. Contact lenses. Despite the extensive use of contact lenses over the last few decades their motion over the eyeball is not yet fully understood, particularly the mechanism by which a lens centers itself over the cornea. An understanding of lens behavior is important in their design, since a poorly fitted lens may lead to corneal problems, also because the fluid motion under the lens controls oxygen diffusion into the eye. The problem is depicted in Figure 4.

In [47] a model is developed with the specific aim of describing the motion of a lens on a tear film; however, the model is applicable to the motion of any object on the surface of a thin film. In two dimensions the Landau-Levich equation (3) is obtained to model tear film thickness around the lens; a three-dimensional version is also derived. The simplifying assumptions made in this analysis, in particular that the eyeball is flat and the lens has constant velocity, mean that the model cannot explain centration, but it does provide a starting point for more complex models and geometries. For example, the two-dimensional model may be easily extended to include variable velocity and rotation relevant to a lens given some initial impulse 


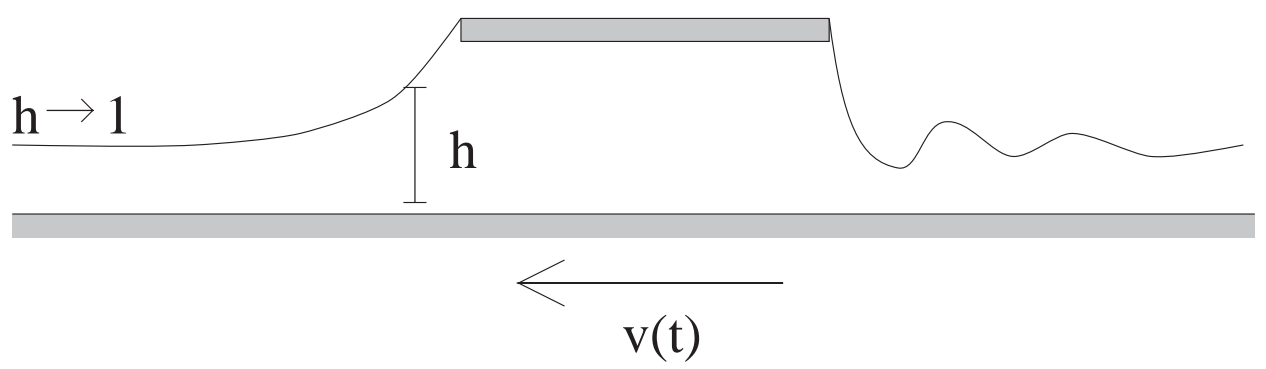

FIG. 4. Contact lens on a thin fluid film.

(from a blink say) and then slowing down. The equation for film height is then

$$
\frac{\partial h}{\partial t}=-\frac{\partial}{\partial x}\left(C \frac{h^{3}}{3} h_{x x x}-v(t) h\right),
$$

where the eyeball velocity, $v(t)$, is determined from the equations of motion.

2.4. Paint drying. A newly painted surface normally has an uneven profile, the most familiar example being "brushmarks." In order for the film to level and produce a smooth finish it must be mobile; however, this allows sagging (a downward flow due to gravity) to take place on an inclined surface. Hence, one of the main problems in painting is balancing the desirable property of levelling with the undesirable one of sagging.

In [53] and [66] it is shown that surface tension may drive the levelling process. With a sinusoidally rippled surface, provided the amplitude is small compared to the film thickness, the authors derive an equation which shows that amplitude decreases exponentially with time. These results have proved successful in analyzing the levelling of viscous liquid films and certain paints, but practical experience shows them to be inadequate for solvent-based paints. In particular, results for predicting the maximum wavelength of brushmarks which may level without excessive sag were contradicted by observation. Further, this model is unable to explain "reversal," a process by which peaks in the initial film may become troughs and vice versa.

The paints studied in [54] consist of a resin dissolved in a volatile solvent. Solvent evaporation is known to give rise to surface tension gradients which provide a mechanism for reversal (more will be said on this in section 2.5). The mathematical theory was later clarified and expanded in [36], [74].

The governing equations for a paint consisting of two components (resin and solvent) are

$$
\begin{aligned}
h_{t}+Q_{x} & =-E, \\
(s h)_{t}+(s Q)_{x} & =-E+D\left(h s_{x}\right)_{x},
\end{aligned}
$$

where $s$ is the solvent concentration, $E$ is the nondimensional evaporation rate, $D$ is the nondimensional diffusion coefficient of the solvent, and the flux $Q$ is given by

$$
Q=\frac{h^{3}}{3 \mu}\left(C h_{x x}-\delta B h\right)_{x}-\frac{h^{2}}{2 \mu} M s_{x} .
$$

Unlike previous examples, the viscosity, $\mu$, is not assumed constant. This model has been used successfully to analyze reversal and may also model "picture framing" 
or fat edges, another common problem in the paint industry. Note that, when no evaporation takes place, $E=0$, the solvent concentration remains constant, and (13) reduces to a form of (1) including gravity on a horizontal plane.

For a review of other problems and defects in paint films, such as craters, pinholes, and bubbles, see [39].

2.5. Marangoni effects. If a free liquid film is to survive for any length of time it must contain surface active agents or surfactants. Hence, pure water will not foam while contaminated water (containing detergent, for example) will.

Surfactants are molecules that preferentially accumulate at a fluid surface. It is energetically favorable for them to occupy the surface rather than the bulk fluid so they reduce surface energy or surface tension. Surfactants give stability to films by two processes [1] which may be understood by considering a finite volume of liquid. If the surface expands, then, in equilibrium, the surface concentration of surfactant will have decreased and the surface tension will therefore be higher. This is known as the Gibbs effect or Gibbs elasticity. Before reaching equilibrium stretching the surface will lead to an even lower concentration of surfactant molecules at the surface. The concentration will increase as molecules move to the surface through the bulk fluid, but until equilibrium is reached the surface tension will be even higher than expected from Gibbs elasticity. This is known as the Marangoni effect. Hence, both the Gibbs effect and the Marangoni effect produce a force which acts to return the surface to its previous state, thus providing stability. Fluid films in general are destabilized by suction forces from the film junctions and gravity drainage. Hence, in the absence of evaporation, the stability of a film depends on the interplay between surface tension and drainage [40].

The Marangoni effect has recently been exploited in a process known as Marangoni drying. This method of drying allows hydrophilic surfaces to be withdrawn from water virtually dry [44], [46]. It is used, for example, in the electronics industry to dry silicon wafers which, after etching with acid, are rinsed in a water bath. When the wafer is withdrawn it retains a thin film of water which might be dried off by traditional methods, such as evaporation or spin drying. However, if the withdrawal takes place in the presence of alcohol vapor, the entrained film can be pulled back into the water.

Marangoni drying relies on an airborne vapor which is water soluble and lowers the surface tension of the water. The water layer thickness is position dependent and, since the thickness is greatest close to the bath, the surface tension is greatest there, as it will have a lower relative concentration of solute. Thus a surface tension gradient is set up which acts to pull the water back into the bath. The basic mechanism can be demonstrated experimentally by placing a piece of cotton wool soaked in alcohol above a thin water film which induces a flow away from the alcohol source. This process has been modelled in [52], where the leading order problem reduces to

$$
\frac{\partial h}{\partial t}=\frac{\partial}{\partial x}\left(\frac{1}{2} M \sigma_{x} h^{2}\right),
$$

where $\sigma$ is the surface tension.

The Marangoni effect can also be used to enhance spin drying. In the presence of alcohol the thin film breaks up into "contracted" droplets (with a contact angle $>90^{\circ}$ ) which are more easily spun off than the original film.

The obvious advantage of Marangoni drying is the processing speed. In the final stages of conventional spin drying the film thickness decays exponentially, so the final layer must be removed by evaporation. This inevitably takes longer than the 
equivalent Marangoni system. An added advantage of this process is that it leaves a clean surface. If the bath water is not scrupulously cleaned, contaminants are left on the surface after evaporation, but when the water is pulled back into the bath the contaminants go with it.

2.6. Long range molecular forces. In reality, a thinning film will often reach a characteristic critical thickness, typically a few hundred angstroms, and then rupture on a very short time-scale. Over such small length-scales long range molecular forces become significant and these have been proposed as a mechanism for this rapid breakdown of a film [62], [71].

The London-van der Waals force is an attractive intermolecular force which will act to thin a film. To model this force a potential energy function/unit liquid volume, $\phi$, was introduced in [59]. This introduces a body force $F=-\nabla \phi$ in the Navier-Stokes equations and leads to

$$
h_{t}+\frac{\partial}{\partial x}\left(C \frac{h^{3}}{3} h_{x x x}+A \frac{h_{x}}{h}\right)=0
$$

for a system driven by surface tension and long range molecular forces.

In [59] the problem of whether a small free surface perturbation grows or decays in the presence of the van der Waals force is investigated. This leads to estimates for the rupture time of a thin film on a solid surface proportional to $h^{5}$ and also for a free film proportional to $h^{3}$. To test the validity of this linear theory equation (17) is re-examined under the influence of nonlinear perturbations in [72]. Their numerical results show the evolution of a film with a slow initial draining followed by a rapidly developing narrow trough in which rupture occurs. Rupture is caused by shorter wavelengths than in the linear analysis, and the nonlinear terms significantly accelerate the speed of rupture.

Assuming the term $\left(h_{x} / h\right)_{x}$ is unrealistic at a microscopic length-scale $(<100 \AA)$ a modified van der Waals term is considered in [16], where a scaled equation for film height, in the vicinity of a contact line, is studied

$$
h_{t}+\frac{\partial}{\partial x}\left(h^{3} h_{x x x}\right)+\frac{\partial^{2}}{\partial x^{2}}\left(h^{m}\right)=0 .
$$

Combining analytical and numerical work they show that (18) permits solutions with $h \rightarrow 0$ and finite speed of propagation at the front.

Ultra-thin films may have a thickness dependent surface tension, introducing Marangoni effects. Further complications, such as thermal effects, condensation, and evaporation, may be introduced. These are included in the model given in [20] which shows numerical solutions for the rupture of a thin film governed by a generalized version of (17).

2.7. Foams and free films. A dry foam is a two-phase fluid consisting of gas separated by a thin continuous liquid film, where the volume fraction of the liquid phase is small. The majority of the liquid is contained in the film junctions or Plateau borders; the films have negligible thickness and liquid content.

When modelling a free film, standard lubrication theory leads to difficulties, since zero shear on the free surface and symmetry at the center of the film provide two conditions to determine a single arbitrary constant in the expression for velocity gradient. One way to avoid this is by taking a higher order approximation such as (6). Alternatively, the difficulty may be overcome by assuming the free surface is saturated 
with surfactant and is therefore inextensible (i.e., to have constant velocity). In [63] this method is used to describe the steady-state for the transition region between the thin film and the plateau border. Since the problem is equivalent to that of dip coating, the method of [42] may be used to show the film thickness is proportional to $C^{2 / 3}$.

Fundamental to the understanding of foam flow in pipes and pores is the motion of a single soapfilm in a pipe. This is modelled experimentally by the soapfilm meter in [2]. In this work an equation for the shape of a static film dependent on gravity, surface tension, and the pressure jump across the film is derived by substituting for curvature, $\kappa$, and hydrostatic pressure in the Laplace-Young equation

$$
p=-\sigma \kappa .
$$

This permits calculation of the critical radius for which a soapfilm can remain intact indefinitely, the result agreeing with the numerical results of [23] on the stability of a liquid column in an inverted cylinder.

Results for foams and soapfilms are also applicable in the study of liquid-liquid emulsions where two droplets coalesce after the fluid separating the droplets has drained and ruptured in the same manner as a soapfilm might. Work on bubble bursting is also relevant, since a bubble about to break through a surface will be separated from the outer phase by a thin liquid film. Indeed, [21] and [31] give results for factors affecting breakthrough in keeping with work on soapfilms. In particular, the time taken for a bubble to rupture a surface is asserted to decrease with the following:

i) bubble diameter decrease;

ii) decrease in the viscosity of the draining film;

iii) interfacial tension increase;

iv) increase in the strength of the van der Waals force;

v) density difference between liquid and air (bubble) decrease.

2.8. Summary. The previous section highlights the wide range of situations in which surface tension drives a thin film flow. The shear flow situation, where one fluid surface is governed by a no-slip condition, is a typical model for coating flows but can also be applied to the movement of condensate on a heat fin and the motion of contact lenses. Free films, such as a single soapfilm, have two free surfaces but the governing equation may be reduced to that of shear flow if one or both of these surfaces are saturated with surfactant. Otherwise higher-order approximations to the Navier-Stokes equations, such as (6), are required.

Allowing surface tension to vary introduces the Marangoni effect. This has been exploited to enhance drying processes, and it also helps in giving stability to foams and soapfilms. In paint drying it will aid the process of surface smoothing, but may lead to "reversal" of the peaks and troughs.

As a film thins and approaches rupture, intermolecular forces must, at some stage, become significant. Equations, such as (17) and (18), which include van der Waals terms may then describe the flow.

\section{Analytical methods.}

3.1. General remarks. Analytic work has centered around the generalized lubrication equation

$$
h_{t}+\left(|h|^{n} h_{x x x}\right)_{x}=0,
$$


or, since the film thickness, $h$, is a nonnegative quantity,

$$
h_{t}+\left(h^{n} h_{x x x}\right)_{x}=0 \text {. }
$$

Like (1) it is a fourth-order nonlinear parabolic equation. It is degenerate since the coefficient of the highest derivative tends to zero as $h$ approaches zero. Of particular relevance to the present study of capillary-driven flow is the case $n=3$; however, values of $n<3$ are of interest, for example, with slip models in the vicinity of $h \rightarrow 0$. Another case of practical importance is $n=1$, which describes the thickness of a thin bridge between two masses of fluid in a Hele-Shaw cell [24].

Steady forms of equation (1) are of considerable importance, as demonstrated by the applications of equation (3), mentioned in section 1 , as are steady flows, for example, at a contact line under the assumption of a travelling wave. It is also worth noting that there is an exact steady solution to (21) with compact support,

$$
h= \begin{cases}a-b x^{2} & |x|<\sqrt{a / b} \\ 0 & |x|>\sqrt{a / b} .\end{cases}
$$

One particular question of interest in the study of equation (21) is whether singularities occur (i.e., the film thickness becomes zero). The development of a singularity corresponds to a topology change in the physical system, for example, when a droplet splits or a hole forms, which in turn alters the mathematical formulation (unless an appropriate weak formulation is used).

Equation (21) has been studied subject to a number of boundary conditions. The most common are

$$
h( \pm a)=1, \quad h_{x x}( \pm a)=p_{0} .
$$

These were introduced in [24] in a Hele-Shaw problem when they correspond to fixed endpoints and a constant external pressure which is balanced by capillary pressure, $x \in[-a, a]$. If $p_{0}$ is positive, the result will be a thinning of the neck, if negative, suction will cause the fluid to bulge out.

$$
h( \pm a)=1, \quad h_{x x x}( \pm a)= \pm c_{0}
$$

again the endpoints are fixed, but now fluid is flowing in or out of the region at a fixed rate $\pm c_{0}$.

$$
h_{x}( \pm a)=0, \quad h_{x x x}( \pm a)=0 ;
$$

these were introduced in [11] to allow integration by parts. They are also a particular type of periodic boundary condition

$$
\frac{\partial^{i} h}{\partial x^{i}}(-a)=\frac{\partial^{i} h}{\partial x^{i}}(a), \quad i=0,1,2,3,
$$

and could, for example, describe a periodic array of droplets.

In the following sections results on ordinary differential forms of the lubrication equation are presented first. Results pertaining to evolution problems are presented in the later sections. 
3.2. Asymptotic results. (i) Dip coating. The method developed in [42], to determine the steady film thickness of a deposit on an infinitely long substrate being pulled out of a bath, has been employed in a number of situations [19], [32], [51], [63]. The basic assumption is that the film shape may be determined in the vicinity of the bath, where a thin film approximation is invalid, via the Laplace-Young equation (19) (or alternatively by assuming it to be a circular arc [19], [32]). Far from the bath the film thickness has a constant, unknown value, $h_{\infty}$, and these two regions must then be matched onto the transition region where (3) determines the film shape.

If the $x$-coordinate is directed away from the bath, the relevant asymptotic solution to (3), as $x \rightarrow \infty$, is

$$
h \sim h_{\infty}+a \exp (-\omega x / 2) \cos (\sqrt{3} \omega x / 2),
$$

where $a$ is a constant $\ll 1$ and $\omega$ is the real cube root of $3 U / C h_{\infty}^{3}$ (of the three possible solutions one is sufficient, since one of the two complex roots may be absorbed into an arbitrary choice of origin and the real cube root gives an unbounded solution as $x \rightarrow \infty)$. This solution provides an initial value for numerical integration of (3). Near the bath the numerical solution is matched to that obtained via the Laplace-Young equation and an expression for the constant film thickness, $h_{\infty}$, is then obtained in terms of the physical parameters. In [73] the same result appears as the first term in an asymptotic series

(28) $h_{\infty}=\sqrt{\frac{\mu U}{\rho g}} \frac{1}{\sqrt{1-\cos \alpha}}\left(0.94581\left(\frac{\mu U}{\sigma}\right)^{1 / 6}+\frac{0.10685 \sin \alpha}{1-\cos \alpha}\left(\frac{\mu U}{\sigma}\right)^{1 / 2}+\cdots\right)$,

valid for values of the capillary number $\mu U / \sigma \ll 1$.

(ii) Draining flows. The asymptotic solution of (8) (which describes fluid draining down a vertical wall) as $x \rightarrow-\infty$ is

$$
h \rightarrow 1+a \exp \left(x / 2^{2 / 3}\right) \cos \left(\sqrt{3} x / 2^{2 / 3}\right) .
$$

As with the previous problem, of three possible independent solutions to the linearized version of (8), this is the only one that is physically acceptable. Using (29) as the condition for an initial value solver, it is discovered in [70] that $h$ never reaches zero. Therefore no solutions to (8) exist which completely describe a fluid layer draining down a dry, vertical wall.

Equation (9) includes terms to model a precursor layer of fluid of thickness $\epsilon$. The difficulty encountered as $h \rightarrow 0$ is thus avoided and a unique solution may be found for $0<\epsilon \leq 1$. Nonetheless, numerical problems may occur if $\epsilon$ is less than the grid size and also in the region where $h=O(\epsilon), x \rightarrow \infty$, but this may be overcome by matching in appropriate regions to the solutions of (3) and (10).

In [70], a graphical relation is given between precursor layer thickness and "apparent contact angle." A significant claim made is that the precursor layer may be ignored as long as the contact angle is specified according to this relation.

(iii) Contact lens flows. An investigation of the steady motion of a contact lens moving on a thin viscous tear film shows that equation (3) describes the tear film height [47]. The relevant boundary value problem is studied in [45]. In the upstream region, using the fact that (3) is autonomous and the solution for $h$ is monotonic, it can be shown that a unique solution of (3) exists for every lens/eyeball separation. Downstream of the lens it may be shown that either zero, one, or two solutions can 
exist, depending on the parameter regime. These results can be confirmed using the asymptotic solution as a starting point for an initial value solver to determine the shape of the tear film.

The corresponding system in polar coordinates, describing a circular contact lens, is also investigated in [47]. Pressure then satisfies Laplace's equation, while the free surface again satisfies a fourth-order nonlinear partial differential equation. Due to the added complexity, the asymptotics are only carried out on a simplified system corresponding to a fast moving lens. However, even for this limiting case, the upstream and downstream problems require a separate numerical solution. The results show that fluid downstream of the lens piles up (forming a capillary wave). Moving around the lens, the wave amplitude decreases until the upstream side is reached, where the film thickness decreases monotonically from the lens to a constant value in the far field. The problem for a lens which is not "fast moving" is not tackled.

(iv) Drop spreading. Asymptotic work on the spreading of drops [33], [41] shows that three regions are required to describe the problem if a slip term is included. These are: an outer region, describing flow in the bulk; an inner region, where $h$ is of the same order as the slip length; and an intermediate region requiring an expansion in terms of the logarithm of the slip length.

In [67] an asymptotic solution to (21) is obtained for $0<n \ll 1$. To overcome difficulties with the singular limit $n \rightarrow 0$ the substitution $v=h^{n / 3}, \tau=(3 / n)^{3} t$ is used and a perturbation expansion derived for $v$. The leading order solution may be used to show (21) possesses waiting time solutions. However, as $t$ tends to infinity the asymptotic solutions tend to simple wave solutions, with a dependence $x / t$. Whereas (21) is expected to give similarity solutions involving $x / t^{(n+4)^{-1}}$, so clearly the asymptotic solution is invalid for large time.

3.3. Travelling waves. The draining flows described in section 2.1 are travelling wave forms of various unsteady lubrication equations, arrived at via the substitution $h(x, t)=H(x-U t)=H(\eta)$ and with the speed of propagation, $U$, set to 1 . Hence, results on draining flows may be interpreted as results for travelling waves.

Substituting the travelling wave form into (21) leads to the third-order autonomous differential equation

$$
H^{n} H_{\eta \eta \eta}=U H+a .
$$

With the appropriate choice of $a, U$, and $n$ this is identical to equation (3). If $a, U$, and $H$ are all positive, then $H_{\eta \eta \eta}>0$. This implies that $H$ can have at most two extrema; a maximum followed by a minimum, as $\eta$ increases. This provides a significant amount of information concerning $H$, for example, to the right of any minimum, $H$, its slope and curvature must increase and $H$ will tend to infinity. To the left of a maximum, $H$ decreases and must reach zero for finite $\eta$. In [17] these properties are exploited to classify the different forms of solution as follows:

- Soliton solutions, with $H$ nonzero over a finite interval;

- Advancing and receding solutions, where the fluid moves into, or away from, a "dry region" (where $H \equiv 0$ );

- Global solutions, where $H>0 \forall \eta$;

- Solutions where touchdown occurs, i.e., $H=0$ at a single point.

Note that if the signs of $a$ and $U$ are different, then these terms may compete and new phenomena, not described by this classification, may arise.

When one of the terms on the right-hand side of (30) is zero, power law behavior near the zeros and infinities of $H$ are given in [17]. Further, with either one of these 
terms zero, the simplified equation is invariant under certain scalings and shift in origin. This indicates that the order may be reduced, leading to a pair of first-order, nonlinear, ordinary differential equations. These are then studied by examining the phase plane trajectories and the results interpreted to determine whether phenomena, such as singularity formation, advancing and receding fronts, and compact support, are permitted for a system.

3.4. Stability analyses. The stability of thin liquid films is important in the final stages of breakup and also in the levelling of uneven ones. In a typical analysis the perturbed film thickness is written

$$
h(x, t)=h_{0}+h_{1}(t) \exp (i \alpha x),
$$

where $\left|h_{1}\right| \ll\left|h_{0}\right|$. Substituting (31) into (21) and linearizing leads to

$$
h_{1} \sim \exp \left(-\alpha^{4} h_{0}^{n} t\right)
$$

so that $h_{1}$ decays exponentially with time and the film is stable. In fact, with periodic boundary conditions it may be shown that a flat interface is stable to linear perturbations [14]. Thus, for small perturbations singularity formation must occur due to external forcing or when the system is far from equilibrium.

Stability analyses on physical systems have been carried out by Pearson [55], who showed that ribbing is stabilized by surface tension effects. Wilson [74] uses the form (31), with $h_{0}=h_{0}(t)$, on a paint drying system to find an integrodifferential equation for $h_{1}$. This is solved asymptotically for a small evaporation rate and numerically to show the perturbation dies away exponentially. In [21] a stability analysis on the thin film between a bubble and a free surface is used to estimate breakthrough times; this leads to the list of factors affecting rupture, given in section 2.7. The linear stability analysis carried out in [37] on steady solutions for a lamella in a capillary tube shows that increasing surface tension or decreasing the applied pressure field stabilizes the lamella.

With a van der Waals term included, a stability analysis on (17) shows that the stability depends on the value of the wave number. When $0<\alpha<\alpha_{c}$, where $\alpha_{c}^{2}=A / C h_{0}^{4}$, disturbances will grow, while for values greater than $\alpha_{c}$ a disturbance will decay [20].

3.5. Similarity solutions. Similarity solutions have been postulated to represent the leading-order behavior for many types of singularity and large time behavior [13], [14]. Since dimensional analysis cannot always predict the time dependence for the similarity variable a general form must be used; for example, singularity formation may be described by

$$
h(x, t)=\tau(t) H\left(\frac{x-x_{p}(t)}{\tau(t)^{q}}\right)=\tau(t) H(\eta),
$$

where $x_{p}(t)$ is the position of the minimum film thickness. The functions $\tau$ and $H$ are subject to the following constraints:

i) $\tau \rightarrow 0$ as the singular time is approached;

ii) $H$ is well behaved for large values of $\eta$; this permits matching at the boundaries;

iii) $H>0$.

Substituting (32) into (21) gives

$$
\frac{\tau_{t}}{\tau}\left(1-q \eta \frac{\partial}{\partial \eta}\right) H-\frac{\dot{x}_{p}}{\tau^{q}} H_{\eta}+\tau^{n-4 q}\left(H^{n} H_{\eta \eta \eta}\right)_{\eta}=0 .
$$


This equation may be reduced to a separable form by choosing $x_{p}=\alpha \tau^{q}$; other simpler forms may be obtained by assuming various terms dominate. The resultant differential equations are the subject of a numerical study into singularity formation in [13] (see section 3.6).

Similarity variables have also been used in the study of (21) for source solutions, i.e., with initial data

$$
h(x, 0)=h_{0} \delta(x),
$$

where $h_{0}$ is the mass. In this case $\tau$ and $q$ may be determined by dimensional analysis to give

$$
h(x, t)=\frac{1}{t^{k}} H\left(\frac{x}{t^{k}}\right)=\frac{1}{t^{k}} H(\eta),
$$

where $k=1 /(n+4)$. Since the source solution is symmetric the resulting equation may be integrated to

$$
H^{n-1} H_{\eta \eta \eta}=k \eta .
$$

This equation is studied in [67] subject to the conditions at the contact line, $\eta=\eta_{1}$,

$$
H\left(\eta_{1}\right)=H^{\prime}\left(\eta_{1}\right)=0,
$$

together with a symmetry condition at the origin. Exact solutions were found for the particular cases $n=0,1$. For $3 / 2<n<3$ approximate solutions were generated using a Frobenius-type series expansion, although it was not shown that these series converged.

In [9] the system (36)-(37), together with the symmetry condition, is proven to have a compactly supported nonnegative solution if and only if $n<3$. This can be seen for $3 / 2<n<3$, since, as $\eta \rightarrow \eta_{1}, H$ takes the form $\left(\eta_{1}-\eta\right)^{\beta}$ [10]; substituting this into (36) and linearizing about $\eta=\eta_{1}$ leads to $\beta=3 / n$. The condition $H_{\eta}=0$ then determines $n<3$.

In [67] an exact solution to (21) is found via separation of variables

$$
h(x, t)= \begin{cases}x^{4 / n}(\lambda n t)^{-1 / n} & x \geq 0, \\ 0 & x \leq 0\end{cases}
$$

where

$$
\lambda=\frac{4}{n}\left(\frac{4}{n}-1\right)\left(\frac{4}{n}-2\right)\left(\frac{4}{n}+1\right) .
$$

This solution is valid for all $n$ except $n=2,4$ and for all values of $t$ such that $\lambda t>0$. For $2<n<4$ both $\lambda$ and $t$ must be negative, indicating the solution will blow up in finite time as $t$ increases to zero. It is postulated that this represents a waiting time solution, an initial redistribution taking place behind a stationary front. Solutions with $n<2$ or $n>4$ behave in the opposite way in that they decay as $t$ increases. When $n=2$ or 4 the equations for the unknown function $X(x)$ are

$$
\left(X^{2} X^{\prime \prime \prime}\right)^{\prime}=-\lambda X, \quad\left(X^{4} X^{\prime \prime \prime}\right)^{\prime}=-\lambda X .
$$

No simple solutions have been found for these equations.

Bernis [8], [9] considers separable solutions to a "modified" lubrication equation

$$
h_{t}+|h|^{n} h_{x x x x}=0 .
$$


This leads to the study of

$$
X^{\prime \prime \prime \prime}=X^{1-n}
$$

for $X>0$ in $(0,1)$ with appropriate smoothness conditions and subject to

$$
X(0)=X^{\prime}(0)=X(1)=X^{\prime}(1)=0 .
$$

This problem may be shown to have a unique solution for $n<4$.

Starov [68] considers the radially symmetric form of the lubrication equation (2) driven solely by surface tension. The similarity substitution $h=t^{-1 / 5} H\left(r / t^{1 / 10}\right)$ transforms it to

$$
\frac{\eta}{10}=H^{2} \frac{\partial}{\partial \eta}\left(\frac{\partial}{\partial \eta}+\frac{1}{\eta}\right) H_{\eta} .
$$

Assuming $h$ is smooth and approximates a spherical cap near the center of the drop it is found there are no solutions of (44) for which $H$ goes to zero for finite $\eta$. However, $H$ does have a minimum and it would appear that the only reasonable conclusion to be drawn from this work is that, as $H$ approaches this minimum, forces other than capillarity (van der Waals, for example) must become significant.

3.6. Unsteady numerical work. In [13] self-similar forms are the subject of numerical investigation into singularity formation. The results are classified according to

i) whether $h \rightarrow 0$ in finite or infinite time,

ii) whether $h \rightarrow 0$ at an interior point or on the boundary,

iii) whether solutions have reflective symmetry about the singular point.

The authors list values of $n$ and the various boundary conditions, (23), (24), and (25), for which these phenomena are observed. Simulations were carried out on various limiting forms of (33) in which different terms dominate. For example, their simulations indicate that the simplest form

$$
H_{\eta \eta \eta}=0
$$

will permit a finite time singularity at the center of the domain for $0 \leq n \leq 1$. The results indicate that the different boundary conditions determine the singularity behavior, while initial conditions determine the location and possibility of a singularity. However, without analytic confirmation, the simulations, which stopped at $h \sim 10^{-15}-10^{-20}$, do not give definite proof of touchdown.

It is well known that the form of solution for (21) may change as $n$ changes. In the numerical work this showed up when the matching conditions at the edge of the similarity region could not be satisfied for certain values of $n$.

In [49] a finite difference scheme was developed to consider coating problems. The authors model a steady draining flow, which has a moving contact line. Comparison of a no-slip model with a particular grid spacing and a slip model with a specified slip length showed exactly the same solution for the correct choice of grid spacing. Hence, it was concluded that grid spacing and slip were equivalent. This method was later incorporated into an unsteady model for the dynamic behavior of a hole in a finite volume of liquid [48]. Whether the hole opens or closes depends upon a competition between surface tension driven levelling, "hoop stress" (through which surface tension acts in the radial plane to close the hole), and the ease with which the contact line may move. Their results show this balance leads to two equilibrium hole radii, one 
stable and one unstable, for each volume of liquid. An analytic investigation of this problem gives similar findings [75].

Coating thickness on a curved substrate (described by equation (7)) is investigated numerically in [64]. In keeping with physical observations, the results show that films thin near external corners and become thicker at internal ones. This is due to an additional, surface tension induced, stress, which occurs as the thin film conforms to the substrate. Hence, at an external corner, the curvature is negative and pulls the fluid down, toward the substrate. At an internal corner, the curvature is positive and has the opposite effect.

Numerical results are also obtained for a coating problem, with a van der Waals force, in [20]. From an initially sinusoidal perturbation the film shape is shown evolving until rupture. In this paper, equation (17) is a particular, isothermal case of a more general equation, allowing for thermal and other effects. Numerical solutions are also obtained for these more complex situations showing, for example, that rupture is delayed by condensation and accelerated by evaporation.

3.7. Mathematical results on (21). A vast amount of analytical work concerns (21), where the results depend not only on the imposed boundary conditions but also on the value of the exponent $n$. In this case it is not possible to do the authors justice and instead the reader will be referred to more appropriate sources.

Results on preservation of nonnegativity are discussed in [11], [13]; preservation of positivity in [13]; singularity formation in finite time [6]; questions concerning the support [6], [11]; finite speed of propagation [7]; nonuniqueness [6]; and the existence of weak solutions [11]. A brief summary of results may be found in [8] and more recently [12]. Other papers worth investigating include [9], [14], [15], [16], and references therein.

3.8. Summary. In this section a number of techniques and results on forms of (1) and (21) have been described. Work on steady forms, such as (3), includes asymptotic results and numerical investigations applied to coating problems, drop spreading, and contact lenses. With the unsteady work, first the travelling wave substitution allows the steady equation to be retrieved. Other techniques include stability analyses, frequently used to estimate rupture times; similarity solutions, which have been postulated to represent the leading order behavior for many types of singularity, to describe source solutions, they also lead to an exact separable solution; and numerical work, particularly on the formation of singularities and the evolution of films in coating flows. Mathematical results, while too numerous to summarize, have also been referenced to allow the reader to research further.

4. Conclusion. Thin film flows driven by surface tension have been shown to describe a diverse range of physical situations and lead to many intriguing mathematical problems. Naturally, a number of these works complement each other. The pioneering work of Landau and Levich [42] paved the way for studies of bubble flow, fluid motion on heat exchangers, soapfilms, and contact lens motion. It is also a particular form of draining flow, as studied in [70], which in turn are travelling wave forms of various unsteady lubrication equations.

Contact line motion, which is still not understood at a basic physical level, may at least be approximately modelled. Standard models introduce a slip length which has subsequently been shown to be equivalent to the grid spacing in a numerical scheme. Models with a precursor film will also lead to identical results. The assumption of a precursor film is also mathematically equivalent to assuming the problem can be 
reformulated in the vicinity of a contact line and matched to a model incorporating molecular forces. This is in keeping with work showing that drop spreading, driven purely by surface tension and with no slip, will not permit a zero film thickness.

A problem akin to contact line motion is that of singularity formation, which also requires $h \rightarrow 0$. Numerical work on purely surface tension driven flows indicates that $h$ may approach zero, without giving definite proof of touchdown. The inclusion of long range molecular forces and surface tension gradients to the numerical models seemingly facilitates the rupture, but again no analytic proof confirms this.

Stability analyses are frequently used to estimate rupture times. However, the analytic results, in general, rely on a linear analysis which will clearly become invalid as the perturbation grows to the order of the film thickness. Numerical studies are therefore still an essential part of modelling this phenomena.

Major gaps in the understanding of thin film flows still exist. Intense investigation is currently underway on the analytic properties of (21); see [12]. However, there are very few analytic studies, or standard mathematical results, on higher dimensional forms such as (2). Foam breakdown and the associated system of governing equations are also poorly understood. The question of the moving contact line will, no doubt, remain for some time and this has significant implications for moving fronts and their stability, for example, in the formation of rivulets.

Finally, it should be pointed out that the ever increasing number of industrial applications of thin film flows and the richness of behavior of the governing equations make this area a particularly rewarding one for mathematicians, engineers, and industrialists alike.

Appendix. Derivation of equation (1). The forces assumed to be driving the thin liquid film, shown in Figure 1, are surface tension, surface tension gradients, gravity, viscous shear, and, for sufficiently thin films (but still thick enough for continuum theory to apply), long range molecular forces. With the coordinate system as shown in Figure 1, equation (1) is derived as follows. First, nondimensionalize length by introducing lengthscales $\left(L, h_{0}\right)$ and velocity scales $(U, \delta U)$, where $L$ is a typical length along the film, $h_{0}$ is a typical film thickness, and $\delta=h_{0} / L \ll 1$. The choice of velocity is determined by the driving force, for example, if surface tension is the sole driving force, then $U$ is chosen to make the inverse capillary number $C=\delta^{3} \sigma_{0} / \mu U=1$, where $\mu$ is the dynamic viscosity and $\sigma_{0}$ is the constant value of surface tension. However, for the present derivation $U$ will remain arbitrary. The pressure scaling $\mu U / \delta^{2} L$ is chosen to balance pressure with viscous forces in (46), thus avoiding a nontrivial solution (in the absence of intermolecular forces) in the limit $\delta \rightarrow 0$.

The leading order terms of the Navier-Stokes equations are now

$$
\begin{aligned}
-(p+\phi)_{x}+u_{y y}+B \sin \alpha & =0, \\
-(p+\phi)_{y}-\delta B \cos \alpha & =0,
\end{aligned}
$$

where $\phi=\phi_{0}+A / h^{3}$ represents the effect of the van der Waals forces (see [27], [59]) and $A$ is related to the Hamaker constant $A^{\prime}$ by $A=A^{\prime} / 6 \pi \delta L^{2} \mu U$. $\phi$ may also be termed as a positive disjoining pressure. While the continuity equation remains unchanged

$$
u_{x}+v_{y}=0 .
$$

The Bond number, $B=\delta^{2} \rho g L^{2} / \mu U$, is a ratio of gravity to viscous forces. In order for the gravity terms to be nonnegligible either $B \sin \alpha$ or $\delta B \cos \alpha$ must be $O(1)$. 
To this order of approximation the boundary conditions on the free surface, $y=h$, are

$$
\begin{aligned}
v & =h_{t}+u h_{x}, \\
p & =-C h_{x x}, \\
u_{y} & =M \sigma_{x} .
\end{aligned}
$$

These represent the kinematic condition, pressure balancing surface tension, and shear stress balancing surface tension gradient. The pressure condition is the LaplaceYoung equation, which reflects the fact that normal stress, due to surface tension, is proportional to curvature. It is standard practice to assume surface tension takes the form $\sigma=\sigma_{0}+\Delta \sigma \sigma_{1}(\mathrm{x})$, where $\sigma_{0}$ is constant while $\sigma_{1}$ varies, hence the Marangoni number $M=\delta \Delta \sigma / \mu U$.

On the substrate, $y=0$, the no-slip condition is

$$
u=v=0 .
$$

Studies involving a moving contact line may often replace $u=0$ with a slip condition, as discussed in section 1 .

Since $\phi$ is a function of $x$ equation (47) can only be linear in $y$ and integration gives

$$
p=-\delta B \cos \alpha(y-h)-C h_{x x} .
$$

Integrating (46) twice and imposing the boundary conditions leads to

$$
u=\left(p_{x}+\phi_{x}-B \sin \alpha\right)\left(\frac{y^{2}}{2}-h y\right)+M \sigma_{x} y .
$$

This may be used in the continuity equation (48) to determine $v$, and, in particular, on the free surface

$$
v(h)=-\int_{0}^{h} u_{x} d y .
$$

This expression together with the kinematic condition leads to the governing equation for film height

$$
h_{t}+Q_{x}=0,
$$

where $Q=\int_{0}^{h} u d y$ is the fluid flux, or equivalently

$$
h_{t}+\frac{\partial}{\partial x}\left\{\frac{h^{3}}{3}\left(C h_{x x x}-\delta B h_{x} \cos \alpha+B \sin \alpha\right)+A \frac{h_{x}}{h}+\frac{M}{2} \sigma_{x} h^{2}\right\}=0 .
$$

Note that, except for approximately horizontal surfaces, where $\alpha \sim O(\delta)$, the gravity term $\delta B h_{x} \cos \alpha$ is negligible in comparison to $B \sin \alpha$.

Acknowledgments. I would like to thank Dr. J. R. Ockendon and Dr. S. D. Howison of Oxford University for motivating this work and providing many helpful comments and also Dr. S. K. Wilson of Strathclyde University, Dr. M. D. Savage and Dr. P. H. Gaskell of Leeds University and Dr. R. Darton of Oxford University for their comments. I gratefully acknowledge financial support from the EPSRC. 


\section{REFERENCES}

[1] J.H. Aubert, A.M. Kraynik, and P.B. Rand, Aqueous foams, Sci. Am., 254 (1986), pp. $58-66$.

[2] M. Barigou and J.F. Davidson, The fluid mechanics of the soap film meter, Chem. Engng Sci., 48 (1993), pp. 2587-2597.

[3] W.D. Bascom, R.L. Cottington, and C.R. Singleterry, In Contact angles, wetability and adhesion, R.F. Gould, ed., Am. Chem. Soc., Washington, 1964, pp. 355-379

[4] H. Benkreira, R. Patel, M.F. Edwards, and W.L. Wilkinson, Classification and analyses of coating flows, J. Non-Newt. Fl. Mech., 54 (1994), pp. 437-447.

[5] D.F. Benjamin and L.E. Scriven, Coating flows: Form and function, Ind. Coat. Res., 1 (1991), pp. 1-37.

[6] E. Beretta, M. Bertsch, and R. Dal Passo, Nonnegative solutions of a fourth order nonlinear degenerate parabolic equation, Arch. Rational Mech. Anal., 129 (1995), pp. 175-200.

[7] F. BERnis Finite speed of propagation for thin viscous flow when $2 \leq n<3$, Comptes Rendus Acad. Sci. I - Math., 322 (1996), pp. 1169-1174.

[8] F. Bernis, Viscous flows, fourth order nonlinear degenerate parabolic equations and singular elliptic problems, Free Boundary Problems, Pitman Res. Notes Math. Ser. 323, Longman Sci. Tech., Harlow, 1995, pp. 40-56.

[9] F. BERnis, On some nonlinear singular boundary value problems of higher order, Nonlinear Anal., 26 (1996), pp. 1061-1078.

[10] F. Bernis, L.A. Peletier, And S.M. Williams, Source type solutions of a fourth order nonlinear degenerate parabolic equation, Nonlinear Anal., 18 (1992), pp. 217-234.

[11] F. Bernis And A. Friedman, Higher order nonlinear degenerate parabolic equations, J. Differ. Equations Appl., 83 (1990), pp. 179-206.

[12] A.L. BertozzI, Lubrication approximations for surface tension driven interfaces: Some open problems, ZAMM, 76 (1996), pp. 373-376.

[13] A.L. Bertozzi, M.P. Brenner, T.F. Dupont, and L.P. Kadanoff, Singularities and similarities in interface flows, in Trends and Perspectives in Appl. Math., Appl. Math. Sci. 100, Springer, New York, 1993, pp. 155-208

[14] A.L. BertozzI, Symmetric singularity formation in lubrication-type equations for interface motion, SIAM J. Appl. Math., 56 (1996), pp. 681-714.

[15] A.L. Bertozzi And M. Pugh, The lubrication approximation for thin viscous films: Regularity and long time behaviour of weak solutions, Comm. Pure Appl. Math., 49 (1996), pp. 85123.

[16] A.L. Bertozzi And M. Pugh, The lubrication approximation for thin viscous films: The moving contact line with a "porous media" cut off of van der Waals interactions, Nonlinearity, 7 (1994), pp. 1535-1564.

[17] S.F. Boatta, L. Kadanoff, And P. Olla, Travelling wave solutions to thin film equations, Phys. Rev. E, 48 (1993), pp. 4423-4431.

[18] M. Brenner And A.L. Bertozzi, On the spreading of droplets on a solid surface, Phys. Rev. Lett., 71 (1993), pp. 593-596.

[19] F.P. Bretherton, The motion of long bubbles in tubes, JFM, 10 (1961), pp. 166-188.

[20] J.P. Burelbach, S.G. Bankoff, and S.H. Davis, Non-linear stability of evaporating/condensing liquid films, JFM, 195 (1988), pp. 463-494.

[21] J-D. Chen, P-S. Hahn, And J.C. Slattery, Coalescence time for a small drop or bubble at a fluid-fluid interface, AIChE J., 30 (1984), pp. 622-630.

[22] K.R. Chun and R.A. Seban, Heat transfer to evaporating liquid films, Trans. ASME J. Heat Transfer, 93 (1971), pp. 391-396.

[23] P. Concus, Static menisci in a vertical right circular cylinder, JFM, 34 (1968), pp. 481-485.

[24] P. Constantin, T.F. Dupont, R.E. Goldstein, L.P. Kadanoff, M.J. Shelley, and S-M. Zhou, Droplet breakup in a model of the Hele-Shaw cell, Phys. Rev. E, 47 (1993), pp. $4169-4181$.

[25] E.B. Dussan V., On the spreading of liquids on solid surfaces: Static and dynamic contact lines, Ann. Rev. Fl. Mech., 11 (1979), pp. 371-400.

[26] E.B. Dussan V. AND S.H. Davis, On the motion of a fluid-fluid interface along a solid surface, JFM, 65 (1974), pp. 71-95.

[27] T. ERneux and S.H. Davis, Nonlinear rupture of free films, Phys. Fl. A, 5 (1993), pp. 11171122.

[28] A. FriEdman, Unresolved mathematical issues in coating flow mechanics, Mathematics in Industrial Problems, IMA Vol. Math. Appl. 16, Springer, New York, 1988. 
[29] P.H. Gaskell, M.D. Savage, J.L. Summers, and H.M. Thompson, Modelling and analysis of meniscus roll coating, JFM, 298 (1995), pp. 113-137.

[30] S. Goldstein, Modern Developments in Fluid Dynamics, Dover, New York, 1965, pp. 676-680.

[31] P-S. Hahn, J-D. Chen, ANd J.C. Slattery, Effects of London-van der Waals forces on the thinning and rupture etc., AIChE J., 31 (1985), pp. 2026-2038.

[32] S. Hirasawa, K. HiJikata, Y. Mori, AND W. NAKAyama, Effect of surface tension on condensate motion in laminar film condensation. (Study of liquid film in a small trough), Int. J. Heat Mass Trans., 23 (1980), pp. 1471-1478.

[33] L.M. Hocking, Rival contact-angle models and the spreading of drops, JFM, 239 (1992), pp. $671-681$.

[34] P.D. Howell, Models for thin viscous sheets, Euro. J. Appl. Math, 7 (1996), pp. 321-343

[35] P.D. Howell, Surface-tension-driven flow on a moving curved surface, JFM, submitted.

[36] S.D. Howison, J.A. Moriarty, J.R. Ockendon, E.L. Terrill, and S.K. Wilson, A mathematical model for drying paint layers, J. Engrg. Math., 32 (1997), pp. 377-394.

[37] M.P. Ida And M.J. Miksis, Dynamics of a lamella in a capillary-tube, SIAM J. Appl. Math., 55 (1995), pp. 23-57.

[38] O.E. Jensen, The thin liquid lining of a weakly curved cylindrical tube, JFM, 331 (1997), pp. 373-403.

[39] L.O. Kornum and H.K.R. Nielsen, Surface defects in drying paint films, Prog. in Org. Coatings, 8 (1980), pp. $275-324$

[40] A.M. Kraynik, Foam flows, Ann. Rev. Fl. Mech., 20 (1988), pp. 325-357.

[41] A.A. LACEY, The motion with slip of a thin viscous droplet over a solid surface, Stud. Appl. Math., 67 (1982), pp. 217-230.

[42] L. Landau And B. Levich, Dragging of a liquid by a moving plate, Acta Physicochim., URSS, 17 (1942), pp. 42-54.

[43] L. Leger and J.F. Joanny, Liquid spreading, Rep. Prog. Phys., (1992), pp. 431-486.

[44] A.F.M. Leenahrs, J.A.M. Huethorst, and J.J. van Oekel, Marangoni drying: A new extremely clean process, Langmuir, 11 (1990), pp. 1701-1703.

[45] J.B. MACLEOD, Solution of a contact lens problem, Free Boundary Problems, Pitman Res. Notes Math., Longman Sci. Tech., Harlow, 1993.

[46] J. Marra And J.A.M. Huethorst, Physical principles of Marangoni drying, Langmuir, 7 (1991), pp. 2748-2755.

[47] J.A. Moriarty AND E.L. Terrill, Mathematical modelling of the motion of hard contact lenses, Euro. J. Appl. Math., 7 (1996), pp. 575-594

[48] J.A. Moriarty And L.W. Schwartz, Dynamic considerations in the opening and closing of holes in thin liquid films, J. Coll. Interf. Sci., 161 (1993), pp. 335-342.

[49] J.A. Moriarty AND L.W. Schwartz, Effective slip in numerical calculations of moving contact-line problems, J. Engrg. Math., 26 (1992), pp. 81-86.

[50] T.G. Myers, Surface tension driven thin film flows, in The Mechanics of Thin Film Coatings, Wiley, 1996

[51] K.J. Mysels, K. Shinoda, and S. Frankel, Soap Films: A Study of Their Thinning and A Bibliography, Pergamon Press, 1959.

[52] S.B.G.M. O'BRIEN, On Marangoni drying: Nonlinear kinematic waves in a thin film, JFM, 254 (1993), pp. 649-670.

[53] S.E. Orchard, Surface levelling in viscous liquids and gels, Appl. Sci. Res. A, 11 (1962), pp. 451-464.

[54] W.S. OverdieP, The levelling of paints, Prog. in Org. Coat., 14 (1986), pp. 159-175.

[55] J.R.A. PEARSON, The instability of uniform viscous flow under rollers and spreaders, JFM, 7 (1960), pp. 481-500.

[56] L. Prandtl, Essentials of Fluid Dynamics, Blackie, London, 1952.

[57] S. REes, An Experimental and Theoretical Investigation of Gravure Roll Coating, Ph.D. thesis, Dept. of Applied Math. Studies, University of Leeds, 1995.

[58] B. Reisfeld, S.G. BAnkoff, And S.H. Davis, The dynamics and stability of thin liquid films during spin coating I Films with constant rates of evaporation or absorption, J. Appl. Phys, 70 (1991), pp. 5258-5277.

[59] E. Ruckenstein AND R.K. Jain, Spontaneous rupture of thin liquid films, Chem. Soc. Faraday Trans. 2, 70 (1973), pp. 132-147.

[60] K.J. Ruschak, Coating flows, Ann. Rev. Fl. Mech., 17 (1985), pp. 65-89.

[61] M.D. Savage, Mathematical model for the onset of ribbing, J. Am. Inst. Chem. Eng., 30 (1984), pp. 999-1002.

[62] A. Scheludko, Thin liquid films, Adv. Coll. Interf. Sci., 4 (1967), pp. 391-464. 
[63] L.W. Schwartz And H.M. Princen, A theory of extensional viscosity for flowing foams and concentrated emulsions, J. Coll. Interf. Sci., 118 (1987), pp. 201-211.

[64] L.W. Schwartz And D.E. Weidner, Modelling of coating flows on curved surfaces, J. Engrg Math., 29 (1995), pp. 91-103.

[65] Y.D. Shikmurzaev, Mathematical modelling of wetting hydrodynamics, Fluid Dynam. Res., 13 (1994), pp. 45-64.

[66] N.P.D. Smith, S.E. Orchard, And A.J. Rhind-Tutt, The physics of brush marks, J. Oil Col. Chem. Assoc., 44 (1961), pp. 618-633.

[67] N.F. Sмyтн And J.M. Hill, High-order nonlinear diffusion, IMA J. Appl. Math., 40 (1988), pp. $73-86$.

[68] V.M. Starov, Spreading of droplets of nonvolatile liquids over a flat solid surface, Coll. J. USSR, 45 (1983), pp. 1009-1015.

[69] L. TANneR, The spreading of silicone oil drops on horizontal surfaces, J. Phys. (D), 12 (1979), pp. $1473-1484$.

[70] E.O. Tuck And L.W. Schwartz, A numerical and asymptotic study of some third-order ordinary differential equations relevant to draining and coating flows, SIAM Rev., 32 (1990), pp. 453-469.

[71] A. VRIJ, Possible mechanism for the spontaneous rupture of thin free liquid films, Disc. Farad. Soc., 42 (1966), pp. 23-33.

[72] M.B. Williams and S.H. Davis, Nonlinear theory of film rupture, J. Coll. Interf. Sci., 90 (1982), pp. 220-228.

[73] S.D.R. WiLson, The drag-out problem in film coating theory, J. Engrg. Math., 16 (1982), pp. 209-221.

[74] S.K. Wilson, The levelling of paint films, IMA J. Appl. Math., 50 (1993), pp. 149-166.

[75] S.K. Wilson AND E.L. TERRILL, The dynamics of planar and axisymmetric holes in thin fluid layers, Phys. Fl., submitted. 